\title{
Nudos en el acompañamiento y la formación en las Empresas de Inserción: agenda de temas pendientes
}

\author{
Fernando Marhuenda Fluixá \\ Natxo Martínez-Rueda \\ Igone Aróstegui Barandica \\ Javier Galarreta Lasa
}

RESUMEN: Las Empresas de Inserción son organizaciones complejas que afrontan diversos desafíos para lograr sus objetivos económicos y sociales. En el contexto español, han experimentado un desarrollo notable en los últimos años, en la medida que se están mostrando como un programa de inserción sociolaboral eficaz para responder a necesidades complejas de inserción laboral.

En este artículo, adoptando un enfoque holístico, se describen las prácticas de formación y acompañamiento y su relación con otras dimensiones del funcionamiento de las El. A partir de ello, se identifican los principales retos que tanto profesionales como investigadores tienen que afrontar para mejorar la empleabilidad de las personas trabajadoras de inserción. Para ello, se ha realizado un análisis de contenido de los informes de investigación de $10 \mathrm{EI}$ del estado español que, resultado de un proceso de observación no participante y entrevistas en profundidad, han elaborado una pareja de investigadores y que han sido validados por cada empresa.

Como resultados, se identifican, en primer lugar, diferentes factores organizacionales que influyen en el desarrollo de los procesos de acompañamiento y cualificación, entre los que destacan las relaciones que la El mantiene con el mercado de trabajo ordinario y las diferentes formas en las que se organizan los procesos productivos. En segundo lugar, se describen las características de los procesos de acompañamiento, analizando sus niveles de estructuración y las fases en las que se articula. En tercer lugar, con relación a los procesos formativos se describen sus diferentes modalidades, con especial referencia a la conciencia y rol de las personas trabajadoras de inserción. Este análisis multidimensional, sirve de base para formular la agenda de temas que tanto investigadores como profesionales tienen que considerar en los próximos años.

PALABRAS CLAVE: Empleabilidad, empresas de inserción, acompañamiento, formación.

CLAVES ECONLIT: I38, L31, M53, P31.

Cómo citar este artículo / How to cite this article: MARHUENDA, F., MARTíNEZ-RUEDA, N., ARÓSTEGUI, I. \& GALARRETA, J. (2020): "Nudos en el acompañamiento y la formación en las Empresas de Inserción: Agenda de temas pendientes", CIRIEC-España, Revista de Economía Pública, Social y Cooperativa, 98, 247285. DOI: 107203/CIRIEC-E.98.16124.

Correspondencia: Fernando Marhuenda Fluixa, Catedrático de Didáctica y Organización Escolar, Universidad de Valencia, fernando.Marhuenda@uv.es; Natxo Martínez-Rueda, Profesor Titular de Pedagogía Social y Diversidad, Universidad de Deusto, natxo.martinez@deusto.es; Igone Aróstegui Barandica, Profesora doctora adjunta, Didáctica y Organización Escolar, Universidad del País Vasco, igone.arostegui@ehu.es, y Javier Galarreta Lasa, Profesor titular de Didáctica y Organización Escolar, Universidad del País Vasco. Email: javier. galarreta@ehu.eus. 


\section{EXPANDED ABSTRACT}

\section{Work Integration Enterprises at the crossroads: An agenda to address the cruxes of accompanying and training processes}

Work integration social enterprises (WISEs) are a type of social enterprise aimed at facilitating access to the ordinary labour market to people at risk of social exclusion. They are transitional structures (SIIS, 2011; Gregg, 2008) given that they attempt to be bridges between the at-risk situation before the labour market and access to employment in an ordinary company. In order to do so, they provide employment in a supported environment, hence giving additional opportunities to develop competencies as well as providing social accompaniment.

The very heart of activities in WISEs, where qualification, production and social accompaniment processes have interplay mutually, turns them into labour contexts featured by hybrid rationales, a mix of productive and social organizations (Campi, Defourny \& Grégoire, 2006; Quintão, 2007; Doherty, Haugh, \& Lyon, 2014; Adam et al., 2017). This, together with the importance of local contexts (Glémain, 2018) contribute to a strong idiosyncratic character of every WISE.

Even if research has identified positive impact of WISEs in terms of access to employment, retaining employment and improving support networks as well as professional competencies, less attention has been paid to the processes through which these results are achieved (Hiu-Kwan Chui, Y Shum, \& S Lum, 2019). Nevertheless, literature points to an interdependence among the different levels and dimensions of the dynamics of WISEs (Adam et al., 2017; Martínez-Rueda, Galarreta, \& Arostegui, 2018). It is therefore relevant to identify the dimensions and factors that influence work processes, foster learning and support, contribute to improve employability and facilitate transition into the ordinary labour market.

\section{Aims}

This article attempts to identify and describe the key dimensions and practices that WISEs develop in their effort to improve employability of their workers with insertion contracts. In order to do so, the article has the following aims:

- Identifying dimensions or key factors around which accompanying and training processes are arranged.

- Describing organizational practices that WISEs develop in their attempt to foster employability.

- Featuring training and accompanying processes in these hybrid and transitional structures. 
- Suggesting in the shape of an agenda the key issues that WISEs have to address in the coming years.

\section{Methodology}

This paper is framed within the literature around case studies that analyze in qualitative ways success factors behind WISEs (Adam et al., 2017). We have performed content analysis of the research reports prepared by couples of researchers after their observations in WISEs, adapting the model of learning trajectories (Eraut, 2009, Marhuenda, 2018). We also conducted interviews to workers with integration contracts as well as to their support workers, as well as two questionnaries of employability, CUFEE (Martínez-Rueda, Aróstegui \& Galarreta, 2018) and CEMI (Llinares, Zacarés \& Córdoba, 2016).

\section{Results}

We have grouped results in three sections. First, as a consequence of describing WISEs as hybrid organizations, we have identified several organizational factors that have an impact upon the development of accompanying and qualification processes: type of relation between the WISE and its promoting institution, the kind of relations with the companies in the same occupational sector, the different kinds of organization of productive activities as well as the features of the workers with integration contracts and the support staff.

Second, we describe the features of the accompanying processes upon analysis of their structuration levels and the stages around which it is developed. We have not only shown the diversity of shapes that accompanying takes in a variety of WISEs, but we have also provided evidence of the links between the levels of structuration and the degree of awareness of workers in integration processes. In relation to the stages, we have found a better definition of access and transition phases rather than the longer developmental phase in-between.

Third, training processes are described in their variety of types and after the factors affecting them. As an example, work-based learning is strongly conditioned by the training potential of the job and the availability of training support. Furthermore, we have identified the participation in processes of formal learning and accreditation as a key element to improve employability. Finally, we have analyzed the influence of the awareness and role of integration workers.

Our paper provides a multidimensional analytical framework to address productive, training and accompanying processes of WISEs in an integrated way, identifying some key factors that may increase the employability of integration workers. 


\section{Conclusions, cautions and contributions of the article}

According to our findings, we dare to give some conclusions and implications both for professionals as well as for researchers that can be considered in order to improve the results of WISEs:

- Need of multidimensional approaches in the analysis of accompanying and qualification practices.

- Relevance of networks and cooperation mechanisms of promoting institutions and ordinary companies with training resources, as key determinants of accompanying and qualification processes.

- Considering the productive organization as a matrix to design accompanying and qualification processes.

- The main variables with an impact upon the training ability of WISEs are the following: reference to a qualification within the National Catalogue of Professional Qualifications, processes of cooperative and teamwork, levels of required production (with a role that can be either facilitating or hindering), and diversity of job positions and spaces.

- Need to develop a reliable and valid system to assess employability that contributes to standardize the process of selection, as well as the evaluation and impact of WISEs.

- Need to design an educational model of its own that, relying upon reference to the job, is able to develop a variety of strategies to assure a qualification matching the existing ones in the ordinary labour market.

- Such an educational model needs to include to key references: occupational profiles linked to jobs and systematization of support upon training routines associated to the professional staff development.

- The educational model needs to be supplemented by training processes on-the-job with participation in formal accreditation processes, to enhance training in the workplace.

The limitations of our research are related to the size of the sample. Despite the variety of productive sectors portrayed, the fact that one of them -recycling-is overrepresented (as so it is among Spanish WISEs), invites us to confirm our results in other sectors.

Summarizing, the article has presented a comprehensive model of the management, accompanying and training processes in WISEs that, taking into account their complexity and diversity, allows us to articulate and relate their different dimensions as the necessary basis to consider a future that is becoming more demanding.

KEYWORDS: Employability, work integration social Enterprise, training, accompanying. 


\section{Introducción}

Las Empresas Sociales dirigidas a la inserción profesional de colectivos desfavorecidos vienen siendo un instrumento frecuente de las políticas públicas de lucha contra el desempleo y la exclusión (Davister, Defourny, \& Grégoire, 2004, Quintão, 2007). En el contexto de mercados laborales cada vez más flexibles y polarizados, estas empresas se configuran en la práctica como realidades heterogéneas y complejas, caracterizadas por la diversidad donde coexisten una amplia variedad de modalidades (Associação A3S, 2014; Adam et al., 2017). La existencia de objetivos múltiples, las diferentes normativas que las regulan, la variedad de agentes con los que se relaciona o las condiciones socioeconómicas específicas de los contextos locales en los que se mueven (Kerlin, 2013, Quintão, 2007) son algunos de los factores que inciden en esa diversidad.

En el contexto español, además de otras modalidades dirigidas a trabajadores con discapacidad, se han regulado las llamadas Empresas de Inserción (EI) como una tipología concreta dirigida a facilitar la incorporación al mercado de trabajo ordinario de personas en situación o riesgo de exclusión. En este sentido, podemos hablar de estructuras transicionales (SIIS, 2011; Gregg, 2008), ya que su finalidad es servir de puente entre la situación de exclusión del mercado de trabajo y el acceso a un empleo en la empresa ordinaria. Un reciente monográfico de la revista del Ciriec (Bel, Lejarriaga y Marhuenda, 2018) abordaba procesos educativos y formativos en empresas de economía social y cooperativismo, con una subsección referida a empresas de inserción, con la que quiere entrar en diálogo este artículo, a partir del trabajo de investigación.

Las El proporcionan un empleo en un entorno de apoyo con oportunidades adicionales de desarrollo de competencias, acompañamiento y un proceso explícito de conexión con empresas ordinarias. Es importante recordar que para las personas en exclusión, la mera participación en un empleo transicional, sin componentes formativos y de apoyo tiene efectos muy limitados en su inserción laboral, aunque presente otros beneficios indirectos para problemáticas específicas (Bloom, 2010). En este sentido hay un consenso acerca de la necesidad de desarrollar estrategias de mejora de la cualificación de las personas trabajadoras (Cooney, 2013), al igual que otras estructuras transicionales (Cid \& Martí, 2012) deben desarrollar prácticas de tres tipos: vinculación social, apoyo social y aprendizaje.

La propia esencia de las actividades de las El, donde los procesos de cualificación y acompañamiento social y los procesos productivos se influyen mutuamente, hace que se constituyan como contextos laborales en los que operan lógicas híbridas, mezcla de organizaciones productivas y or- 
ganizaciones sociales (Campi, Defourny \& Grégoire, 2006; Quintão, 2007; Doherty, Haugh, \& Lyon, 2014; Adam et al., 2017).

Este conjunto de factores, junto a su carácter de 'organizaciones territorializadas' (Glémain, 2018) contribuye a que cada El tenga un fuerte carácter 'idiosincrático'. Es decir, cada El articula los diferentes elementos que las componen (actividad productiva, actividad formativa, acompañamiento social, relaciones con el mercado...) de una forma particular, dando lugar a diferentes modelos 0 tipologías de El (Cooney, 2015; Quintão, 2007; Associação A3S, 2014; Marhuenda-Fluixá, 2018; Davister, Defourny \& Gregoire, 2004).

Aunque los estudios han identificado impactos positivos de las El en términos de inserción laboral, mantenimiento del empleo, mejoras de las redes de apoyo o en las competencias profesionales, se ha prestado menos atención a los procesos a través de los cuales se logran estos resultados (Hiu-Kwan Chui, Y Shum, \& S Lum, 2019). Por ello, es relevante identificar las dimensiones o factores que influyen en cómo se desarrollan los procesos de trabajo, aprendizaje y apoyo que contribuyen a mejorar la empleabilidad de las personas y a facilitar la transición al mercado ordinario.

El objetivo de este artículo es identificar las dimensiones de funcionamiento de las El relacionadas con la mejora de la empleabilidad, describiendo las formas específicas que adoptan los procesos de acompañamiento y formación. Se espera con ello formular las cuestiones o temas clave que tanto investigadores como profesionales de las El tienen que considerar en el futuro para mejorar su eficacia.

Este trabajo se enmarca en la literatura sobre estudios de caso que analizan de forma cualitativa los factores de éxito relevantes en las El (Adam et al., 2017).

\section{Estado de la cuestión}

\subsection{Empresas de inserción en España}

La Ley 44/2007 de Empresas de Inserción identifica la mejora de la empleabilidad -como el objetivo de las El con la finalidad de que las personas trabajadoras de inserción puedan acceder a un empleo ordinario. La noción de empleabilidad, que tiene carácter multidimensional y resulta controvertida, se ha abordado en este contexto desde distintas perspectivas (Llinares, Zacarés y Córdoba, 2016; Martínez, Arostegui y Galarreta, 2018). Se señala que 'deberán tener servicios de intervención o acompañamiento para la inserción sociolaboral que faciliten su posterior incorporación al mercado de trabajo ordinario' (Art. 4). 
La oferta de las El se basa en un contrato de trabajo, con un límite de tres años, que tiene que ser complementado con apoyos tales como trabajo asistido, formación en el puesto de trabajo, formación para la adecuación del nivel formativo a las exigencias del mercado, servicios de acompañamiento social y apoyos en la transición al mercado de trabajo ordinario. Además de las personas trabajadoras de inserción (PTI), las El incorporan a personal técnico de apoyo a la producción (PTP) y a personal técnico de acompañamiento (PTA).

En el Estado Español, las El han experimentado un notable desarrollo aunque de forma desigual entre los diferentes territorios (Askunze, 2016). Según datos de la Memoria Social de 2017 (FAEDEI, 2018), se contabilizan 178 El asociadas a FAEDEI con 3438 PTI con una tasa de inserción en el mercado ordinario del $62,3 \%$, que sube hasta el $69,8 \%$ si se consideran las inserciones en las propias El. En la vertiente económica, tuvieron unos ingresos totales de 119,56 millones de euros, de los cuales el $78,81 \%$ proceden de su actividad comercial.

Las El, tal como señala la ley, son creadas por entidades promotoras que en su mayoría son entidades del sector no lucrativo, aunque una pequeña proporción está impulsada por entidades públicas de carácter local. Una visión actual de la situación de las El en España la ofrecen Belassir, Calabozo y Treguer (2019).

La mayor parte de las El se mueven en el sector servicios $(80,99 \%)$ con predominio de la gestión y tratamiento de residuos, el comercio y la hostelería, aunque con una gran diversidad de actividades económicas. Al igual que en otros países (Cooney, 2015), las El se mueven en sectores con bajos niveles de cualificación y salarios siendo en su mayor parte empresas pequeñas que actúan en los mercados locales y con alto grado de heterogeneidad (O'Connor y Meinhard, 2014).

\subsection{Las empresas de inserción, empresas con objetivos sociales}

Como hemos señalado, las El se caracterizan por buscar un equilibrio entre los objetivos sociales y sus objetivos económicos. Esto implica que su organización y funcionamiento se orienta, además de a su viabilidad, a la mejora de la empleabilidad de las personas trabajadoras de inserción, configurándose como organizaciones que funcionan con lógicas híbridas (Battilana, School, Sengul, College, \& Pache, 2015; Martínez \& Molpeceres, 2010; Quintão, 2007). En esta línea, es relevante tener presente que las empresas sociales cuando están expuestas a presiones institucionales y económicas, su atención puede desviarse de los objetivos sociales, generándose situaciones de desequilibrio entre sus diferentes objetivos (Audenaert, Van Der Heijden, Conway, Crucke, \& Decramer, 2019). Por ello, es probable que la situación económica de las empresas tenga un papel relevante en cómo se desarrollan los procesos de acompañamiento y aprendizaje (Martínez-Rueda, Galarreta, \& Arostegui, 2018). 
Aunque son más frecuentes los estudios sobre los factores que influyen en el éxito de las El en su vertiente empresarial que en su vertiente social, se aprecia una interdependencia entre los diferentes niveles y dimensiones del funcionamiento de las El (Adam et al., 2017). Por ejemplo, Martínez-Rueda, Galarreta \& Arostegui (2018) identifican tres dimensiones de las El que contribuyen a la empleabilidad. Por una parte, un conjunto de factores relacionados con el desarrollo técnico y organizativo y sus relaciones con el mercado de trabajo. Una segunda dimensión relacionada con las características formativas de los puestos de trabajo y una tercera que se refiere a los tipos e intensidad de los apoyos disponibles para las personas.

Estos autores señalan las relaciones con el entorno productivo y con el mercado de trabajo como un factor clave en los resultados sociales de las El. En esta línea, Adam et al., (2017) también apuntan a la imagen de la El en los sectores productivos en los que se mueve como una variable clave; así como a su importancia, en tanto que empresas sociales, en relación con las administraciones para promover empleo decente (Álvarez, Cózar, Martínez y Melían, 2019).

\subsection{Las empresas de inserción como contexto de acompañamiento y apoyo}

La variedad de servicios de apoyo a las personas trabajadoras se ha identificado como un factor que contribuye a que las El mejoren la empleabilidad de sus trabajadores de inserción. Esos apoyos deben dirigirse tanto a su incorporación al mercado laboral como a la participación social (Adam et al., 2017). Desde las El se han desarrollado algunos esfuerzos por sistematizar los apoyos que prestan (Martinez-Rueda \& Galarreta, 2019), identificando los siguientes: diagnóstico de empleabilidad, plan personal de empleo, apoyos a la actividad laboral, formación en el puesto, formación interna y externa, acompañamiento social, apoyo al empoderamiento y la participación social, plan de búsqueda activa de empleo en entornos ordinarios y apoyo a la búsqueda y mantenimiento del empleo.

En la intervención social, la prestación de esta variedad de apoyos se ha articulado en torno al concepto de acompañamiento, entendido como una metodología de intervención profesional (Fantova, 2009; Perez Eransus, 2004; Planella, 2008) de carácter relacional, educativa, centrada en la persona y mediadora en el acceso a diferentes recursos y apoyos sociales; tal y como han expuesto también San José, Roca y Castelló (2019).

Esta metodología ha sido adoptado por las El (Fernández, Galarreta \& Martínez, 2007; FAEDEI \& AERESS, 2014; Gallastegi \& Martínez, 2011; Martínez-Rueda, Galarreta \& Aróstegui, 2018) como referencia para la actuación profesional y el desarrollo de itinerarios de inserción sociolaboral. Estos itinerarios se desarrollan, con pequeñas variaciones, en tres grandes fases (Abiétar López, Ros-Garrido, \& Marhuenda Fluixá, 2018): Incorporación (acogida, diagnóstico de empleabilidad y plan personal de empleo), desarrollo (desempeño laboral y acciones de mejora de la empleabilidad) y la fase de salida o transición al mercado ordinario. 
En el desarrollo de estos itinerarios se considera que el establecimiento de expectativas claras y de incentivos contribuyen a que los trabajadores vulnerables puedan anticipar y optimizar sus competencias (Audenaert et al., 2019), mejorando, en consecuencia, su empleabilidad. En ese sentido, las El promueven que las PTI puedan tener un mayor control de su situación vital, con la identificación de sus metas personales y de las acciones que pueden poner en marcha para conseguirlas, con énfasis en el empleo.

\subsection{Las empresas de inserción como entorno de aprendizaje}

Las El trabajan con personas adultas que, salvo excepciones, llevan ya un tiempo largo fuera del sistema educativo formal y con frecuencia carecen de títulos o cualificaciones. En este sentido, un objetivo central de las El es mejorar la cualificación profesional, social y personal de las personas trabajadoras de inserción. Por ello, cabe esperar una cantidad considerable de procesos educativos informales vinculados al desempeño del puesto de trabajo, orientados a que la persona adquiera cada vez más autonomía, adopte más decisiones vinculadas a qué hacer con su vida, su trabajo y sus cualificaciones, así como el reconocimiento de estas (Cebolla, Chisvert-Tarazona y Guillera, 2019).

Para que este avance se materialice, el personal técnico de apoyo mantiene unas relaciones con las PTI que, además de laborales, son también educativas o pedagógicas (Eraut 2009, 2007, 2005, 2004), donde el aprendizaje en el propio puesto juega un papel central. El enfoque de este autor sobre las trayectorias de aprendizaje (Eraut, 2009) ofrece un marco de análisis amplio y con capacidad de ser aplicado a las El. Permite identificar los efectos educativos de los procesos productivos al prestar atención a los contextos y condiciones de trabajo y a los conocimientos y destrezas que se necesitan para el desempeño del puesto de trabajo. Su aproximación nos permite diferenciar entre factores personales (ya sean de las PTI o del personal de apoyo) y factores contextuales que afectan al aprendizaje, al tiempo que permite abordar la interacción que los dos tipos de factores pueden tener entre sí.

Por otra parte, debido al carácter transicional de las El, podemos esperar que cuenten con los rasgos de lo que Evans, Hodkinson, Rainbird y Unwin (2006) han denominado entornos expansivos de aprendizaje o plataformas de transición, que se caracterizan por: la participación en diversos grupos y equipos de trabajo y en entorno más amplios, acceso a oportunidades formativas amplias, transiciones graduales, reconocimiento de la contribución individual, valorización de las competencias técnicas, apoyo y estímulo al progreso personal y rol facilitador del personal responsable. 


\section{Objetivos y metodología}

Como hemos señalado, este artículo pretende identificar y describir las dimensiones clave y las prácticas que desarrollan las El en su esfuerzo de mejorar la empleabilidad de las personas trabajadoras de inserción. Para ello, se propone los siguientes objetivos:

- Identificar dimensiones o factores clave en torno a los cuales se configuran los procesos de acompañamiento y formación de las El.

- Describir prácticas organizacionales que desarrollan las El en su intento de desarrollar la empleabilidad.

- Caracterizar los procesos de formación y acompañamiento de las El, consideradas como estructuras híbridas y transicionales.

- Formular, a modo de propuesta, las cuestiones clave que las El tienen que desarrollar en los próximos años.

Para ello, se han tomado como base los productos de investigación generados en el marco del Proyecto "Procesos de formación, acompañamiento, cualificación y desarrollo personal en empresas de inserción: innovación en la inclusión social desde el empleo" financiado por el Gobierno de España (EDU2013-45919-R), realizado en el periodo de 4 años 2014-2018, y cuya finalidad fue describir la dinámica formativa de las El y ver en qué medida se orientan al aprendizaje y a la promoción personal y social.

En este artículo damos cuenta de parte del trabajo realizado en 10 El de diferentes Comunidades Autónomas de España, de las que se recoge información tanto cuantitativa como cualitativa (Abiétar, Ros-Garrido, Marhuenda, 2018). La selección de las El se realiza de común acuerdo con Federación de Asociaciones de Empresas de Inserción, FAEDEI, y en su caso con entidades autonómicas, quién extiende la invitación a todas las El interesadas en participar, y que cumplen con los siguientes requisitos: a) tener personas trabajadoras de inserción que se encuentren en alguna de las 3 fases del itinerario (fase inicial de acogida, fase intermedia de formación y acompañamiento y fase de salida 0 transición al mercado ordinario); y b) ser una El consolidada. Una vez, se cuenta con las entidades disponibles y que cumplen con los criterios, se procede a seleccionar primando que pueda haber diversidad de empresas, atendiendo a sector productivo, entidades promotoras y personas destinatarias.

El trabajo de campo lo desarrollan dos investigadores por empresa y a través de una observación no participante, describen el desarrollo de una jornada laboral de entre 2 y 4 PTI. Esta observación 
se complementa con entrevistas semiestructuradas en profundidad a las PTI y al personal técnico de apoyo y con las respuestas al Cuestionario de Factores Organizacionales de Empleabilidad-CUFEE (Martínez-Rueda, Aróstegui y Galarreta, 2018) completado por dos profesionales de cada empresa, así como al Cuestionario de Medida de la Empleabilidad Individual-CEMI (Llinares, Zacarés \& Córdoba, 2016). Con toda esa información, cada pareja de investigadores elabora un informe que se remite a cada El para su validación. El guion de este informe es previamente consensuado en el equipo de investigación y recoge los elementos señalados en la tabla 1. En el desarrollo de la investigación se ha llevado a cabo el protocolo ético: solicitud de permisos a las El, tanto profesionales como PTI, consentimientos informados para la recogida de datos en las entrevistas, así como el contraste y triangulación de los informes con responsables de las El participantes.

\section{Tabla 1. Guión informe de Empresas de Inserción (El)}

\begin{tabular}{|l|l|}
\hline Ejes del informe & Elementos \\
\hline La Empresa de Inserción & Contexto general de la El \\
& Entorno socioeconómico \\
& Características de la entidad promotora y de la EI \\
& Características de los puestos de trabajo \\
\hline Las Personas Trabajadoras en Inserción & Caracterización general de las PTI observadas \\
& Qué y cómo se aprende en la El \\
& Aprendizajes que llevan a cabo las PTI \\
& Cómo tiene lugar el aprendizaje en la El \\
& Evolución de los indicadores de empleabilidad \\
\hline Profesionales de apoyo a la inserción & Caracterización de los profesionales de apoyo a la inserción \\
& Proceso de acompañamiento y aprendizaje \\
\hline
\end{tabular}

\subsection{Procedimiento}

En este artículo se presentan los resultados de análisis de estos informes elaborados por cada pareja de investigadores y validados por cada empresa.

El análisis de los informes se realiza con la técnica de análisis de contenido de los 10 informes de las El. Esta técnica resulta de interés para establecer las inferencias o explicaciones en torno a la realidad (Andréu Abela, 2002). En este estudio el análisis del contenido se realiza sobre los informes de una muestra de $10 \mathrm{El}$ de todo el Estado, a partir de la cual se establece una caracterización de tipos de El y su influencia en los procesos formativos y de acompañamiento en las El. Las categorías de análisis se ordenan, en primer lugar, por el título de los apartados incluidos en los informes y recogidos en el guion. En segundo lugar, mediante un análisis más concreto detecta aquella información recurrente, iterativa, o aquella que el investigador ha resaltado o en la que pone su atención de manera especial. 


\subsection{Características de la muestra participante}

Como se puede ver (Tabla 2) de las $10 \mathrm{El}$, el $60 \%$ de las El del estudio desarrollan su actividad productiva en el sector de la recuperación y reciclaje. Tres cuartas partes de la muestra son de la zona este del país, tres de la zona norte y dos del centro del país. Con relación al tamaño de la empresa, destaca que la mayor parte de las empresas son de menos de 50 trabajadores mientras que sólo 2 se sitúan por encima de los 50 trabajadores. En cuanto al colectivo al que se dirigen, aunque en todos los casos son personas en situaciones de riesgo o exclusión, en el $60 \%$ no se dirige a un colectivo específico, sino a una diversidad de situaciones, mientras que tres empresas se dirigen a mujeres y en un caso se orienta a jóvenes en situación de riesgo o exclusión social.

\section{Tabla 2. Muestra del estudio}

\begin{tabular}{|l|c|c|l|l|}
\hline Empresa de Inserción & Fecha creación & $\begin{array}{c}\text { Tamaño } \\
\text { El }\end{array}$ & \multicolumn{1}{|c|}{ Colectivo } & \multicolumn{1}{|c|}{ Sector de Actividad } \\
\hline CENTRO-1 & 2007 & Intermedia & $\begin{array}{l}\text { Mujeres en riesgo 0 } \\
\text { exclusión }\end{array}$ & Hostelería y Servicios \\
\hline CENTRO-2 & 2008 & Pequeña & Diverso & Lavandería Industrial \\
\hline ESTE-1 & 2010 & Pequeña & $\begin{array}{l}\text { Mujeres en riesgo o } \\
\text { exclusión }\end{array}$ & Servicios diversos \\
\hline ESTE-2 & $2013^{1}$ & Mediana & Diverso & Recuperación y reciclaje \\
\hline ESTE-3 & 2005 & Intermedia & Diverso & Recuperación y reciclaje \\
\hline ESTE-4 & 2015 & Intermedia & Diverso & Recuperación y reciclaje \\
\hline ESTE-5 & 2007 & Micro & Mujeres & Recuperación y reciclaje \\
\hline NORTE-1 & 2003 & Mediana & Diverso & Recuperación y reciclaje \\
\hline NORTE-2 & 2002 & Pequeña & Diverso & Recuperación y reciclaje \\
\hline NORTE-3 & 2004 & Intermedia & Jóvenes & Hostelería/Restauración \\
\hline
\end{tabular}

1.- Pese a constituirse como tal en 2013 , se trata de una empresa promovida por otra matriz que está ampliando su presencia en distintas comunidades autónomas, de ahi que la consideremos como una empresa consolidada. 


\section{Resultados}

Hemos organizado la presentación y discusión de los resultados de acuerdo con los objetivos de la investigación en tres niveles. En el primero nivel se contextualizan los procesos de cualificación y acompañamiento de las El, describimos algunas de las variables identificadas en la organización de las El y que tienen influencia en los procesos formativos, así como algunas modalidades en las que se desarrollan esas variables. En el segundo avanzamos aspectos relacionados con los procesos de acompañamiento para centrarnos, en una tercera parte, en los procesos formativos que se desarrollan en dichos contextos.

\subsection{Las El como organizaciones híbridas: Factores organizacionales que condi- cionan el desarrollo de los procesos de cualificación y apoyo}

Las El para cumplir con su fin social de inclusión laboral de personas en situación o riesgo de exclusión se constituyen como organizaciones productivas de bienes o servicios. Por ello, y dado que en primer lugar son estructuras productivas es relevante describir las formas y los condicionantes que les configuran como estructuras empresariales que influyen y condicionan los procesos de cualificación y apoyo: el entorno de la El, la organización del contexto laboral y de los procesos productivos y las personas implicadas en su desarrollo.

\subsubsection{El entorno de la Empresa de Inserción}

Como organizaciones complejas y multiagente (Campi et al., 2006), las El establecen relaciones diversas con múltiples agentes, atendiendo a sus diferentes dimensiones de funcionamiento (económico-productiva, social, institucional...). Del análisis de los informes se derivan dos aspectos clave, la relación de la El con la entidad promotora y las relaciones que mantiene con el sector productivo más amplio en el que se ubica.

\section{Relaciones entre la El y la entidad promotora}

Como se ha señalado, todas las El se crean a partir de una entidad promotora, teniendo que estar participada al menos con el $51 \%$ del capital social por una organización social o entidad sin ánimo de lucro. Estas entidades encuentran en las El la posibilidad de desarrollar su misión a través de crear iniciativas de carácter económico. Son, como el propio tercer sector, entidades diversas en cuanto a sus trayectorias, motivaciones y sectores de actuación, aunque comparten su trabajo con colectivos 
desfavorecidos 0 en situación de exclusión, bien desde los servicios sociales o desde los programas de formación y empleo.

Un primer elemento que se identifica en las descripciones son las relaciones que mantiene la entidad promotora con la El. En el análisis de las empresas de la investigación, identificamos tres modalidades o formas diferentes en las que se establecen relaciones entre la entidad promotora y la El:

a) Integrada. La El forma parte de la estructura de la entidad promotora en los procesos de trabajo, en la organización de los equipos humanos y en la lógica de su funcionamiento. Se muestra con claridad que la El es un eslabón en la oferta de servicios de la entidad, contribuyendo a la creación de itinerarios.

Servicios de Norte-3 está promovida por una cooperativa de iniciativa social que desarrolla programas de formación profesional reglada (Formación Profesional Básica, Grado Medio), Educación de adultos, Formación Profesional para el empleo, programas de orientación y promueve empresas de inserción, como parte de sus itinerarios de inserción laboral. La El se integra en una estructura funcional del sector (hostelería), que desarrolla de una manera integrada los programas formativos como la propia El (Norte-3, p. 5-6).

Se puede dar incluso una integración de recursos humanos entre una y otra Los profesionales pueden desarrollar funciones tanto en la empresa de inserción como en los programas formativos (Norte-3, p. 6).

b) Especializada. La entidad promotora está especializada en crear y gestionar El, siendo esa su misión o actividad principal. Aunque pueda incluir actividades de formación se dirigen a la cualificación del personal, previo a su incorporación a la empresa o como formación continua Este-2 es una El que forma parte de una cooperativa de segundo grado en la que participan Cooperativas de Iniciativa Social y Empresas de Inserción impulsadas por una organización eclesiástica. Su objetivo es la inserción sociolaboral de personas en situación o riesgo de exclusión social, mediante actividades de servicios ambientales, reutilización y reciclaje, consumo sostenible, formación y atención a personas (Este-2, p. 2).

c) Autónoma o paralela. La El es uno de los proyectos o actividades de la entidad promotora que funciona con una cierta autonomía con relación a ella. En este caso, las entidades promotoras desarrollan una variedad de programas de intervención social, uno de los cuales son El.

La El Este-1 es promovida por una asociación que desarrolla programas socio-educativos dirigidos a mujeres, menores y familias en situación de vulnerabilidad social, que pretenden, actuando sobre la salud, educación y tiempo libre, acceso a la vivienda, empleo y convivencia familiar, contribuir a la inclusión social y empoderamiento de cada núcleo familiar. (Este-1, p. 2) 
Un aspecto relacionado, aunque no se identifica con ninguna de las modalidades b o c, tiene que ver con el grado en el que la El y la entidad promotora comparten algunos recursos como, por ejemplo, servicios de orientación laboral o intermediación.

\section{Relaciones que mantiene la El con el sector productivo}

Todas las empresas de inserción como productoras de bienes o servicios se mueven en un determinado sector productivo con el que mantienen diversas relaciones de competencia o colaboración. Atendiendo a las relaciones que mantienen hemos identificado tres modalidades diferentes:

a) Mercados reservados o especializados. Son mercados en los que no se da una competencia con otras empresas (o es de carácter limitado) al no ser una actividad productiva considerada de interés por la iniciativa privada. El ejemplo más claro es el sector de la recuperación y el reciclaje que, en parte, se encuentra apoyado por la iniciativa pública. A pesar de ello, y para añadirle complejidad, hay que puntualizar que puede haber algunas dimensiones de ese proceso (p.e. la comercialización) que sí puede establecer relaciones de competencia con otras El.

Norte-1 tiene dos empresas que se dedican a recuperación de mobiliario, aparatos eléctricos y electrónicos, textiles y otros enseres desechados como residuos y a la comercialización de otros productos solidarios. (Norte-1, p. 5)

b) Mercado competitivo. Muchas El compiten en el mercado en igualdad de condiciones (aunque se hayan incorporado cláusulas sociales) con otras empresas del sector. Empresas de limpieza o buena parte de la hostelería se sitúan en esta modalidad.

Las empresas del sector de la lavandería industrial compiten entre ellas, principalmente, con dos herramientas que repercuten directamente sobre las personas trabajadoras: 1.- La calidad y la velocidad en los tiempos de entrega, lo que exige un alto ritmo de trabajo y un escaso margen de error. 2.- La competencia por precios bajos, lo que hace que las empresas tengan uno de los convenios colectivos más bajos en relación con el salario mínimo interprofesional. (Centro-2, p. 6)

c) Mercado subsidiario o colaborativo. Aunque no se puede hablar de modalidades puras, sí podemos encontrar algunas empresas que mantienen relaciones de colaboración con una 0 varias empresas para las que desarrollan su trabajo. Esta modalidad, frecuentemente utilizada por los centros especiales de empleo, también se da en algunas El de forma puntual, aunque se apunta a su interés

En los dos últimos años ha tratado de ampliar la línea de negocio hacia la tintorería, pero la experiencia de asociación con una empresa tecnológica. (...) Este es un inconveniente común a las empresas de inserción hoy en día en España, no están acostumbradas a establecer y gestionar alianza con otras empresas de las denominadas ordinarias. (Centro-2, p. 5) 
Además de la vertiente productiva, estas diferentes configuraciones tienen un gran impacto en los procesos de formación y cualificación por cuanto definen las competencias profesionales a desarrollar y su encaje en el mercado.

Por otro lado, las El también mantienen relaciones con el tejido empresarial con el objetivo de facilitar la incorporación de las PTI a las empresas. En parte condicionado por lo anterior, pero también teniendo en cuenta otras variables, las El desarrollan un conjunto de actividades de colaboración con esas empresas. Para conseguir su objetivo social tienen que desarrollar actividades de prospección y conocimiento del mercado, de análisis de perfiles y necesidades formativas, de intermediación, etc... Aunque a través del análisis de los informes no hemos podido identificar diferentes estrategias en este aspecto, sí se aprecia una mayor o menor intensidad en el grado en el que las El colaboran con empresas ordinarias para incorporar a PTI. Por ejemplo,

Este-2 contrató a una persona como prospectora de empleo en el año 2016. Encargada de contactar con empresas para facilitar entrevistas de trabajo y oportunidades de empleo para las PTI que se encuentran en la fase de salida al mercado ordinario. (Este-2, p. 19) Las relaciones de la El con el mercado de trabajo son buenas, valorándose muy positivamente la prospección e intermediación laboral (Este-4, p. 4)

Los resultados manifiestan debilidades en relación al seguimiento de la inserción, labores de intermediación, prospección y el establecimiento de contactos con el sector productivo, aspecto que se ha observado a lo largo del trabajo de campo. (Norte-2, p. 7)

\subsubsection{La organización de las actividades productivas}

La actividad económico-productiva de las El es otro de los aspectos centrales que condicionan los procesos formativos y de acompañamiento.

Con relación a la actividad productiva un aspecto que influye en la dinámica de la El es el tipo de sector productivo en el que se mueven. Desarrollar la actividad productiva en un sector determinado (reciclaje, hostelería, limpieza...) tiene implicaciones en la organización del trabajo. Aunque de la pertenencia a un determinado sector no puede derivarse de forma automática que los procesos de trabajo se desarrollen de una determinada manera, sí hemos identificado una influencia importante en la medida en que, según los sectores, se dan algunos patrones de organización del trabajo. Por ejemplo, diferentes empresas del sector de hostelería enfatizan la relevancia que tiene el trabajo en equipo. Es el caso de la hostelería donde

La centralidad que adquiere en el proceso de aprendizaje la conciencia de que el trabajo en la cafetería es un trabajo en equipo. A pesar de los diferentes grados de experiencia y desempeño de las trabajadoras, esta conciencia es clave en el funcionamiento diario de la El (Centro-1, p.2). 
Uno de los puntos fuertes de aprendizaje en esta El es el fomento del trabajo en equipo, de las relaciones sociales, la comunicación interna que se caracteriza por su rotación, movilidad que favorece el trabajo en diferentes grupos humanos según necesidad (horas punta en el servicio de barra, acudir a servir un catering con PTIs y responsable de otros centros de trabajo de la El...) y la relación con clientes (Norte 3, p. 13).

A pesar de que en la mayor parte de las empresas se identifica como un elemento relevante, en otros sectores no adquiere la misma importancia:

No hemos detectado que el trabajo en equipo se fomente explícitamente como un valor, aunque en la práctica la organización de la tienda o el almacén lo reclamen y se desarrolle con cierta naturalidad (Norte-1, 16).

Otra variable identificada tiene que ver con los 'niveles de exigencia productiva', aunque es percibido de forma diferente según empresas. En algunas de ellas, aunque el ritmo de trabajo es un aspecto que aparece como necesario en el funcionamiento de las empresas, el alto ritmo de trabajo se experimenta de forma negativa

deficiencias (...), vienen determinadas por la necesidad de la empresa de mantener un elevado de ritmo de trabajo lo que ponen en peligro la calidad de los procesos de acompañamiento, de formación y de seguimiento de los procesos de inserción (Norte-2, p. 7)

la premura de que la producción salga adelante puede incidir en los procesos y potencialidades de aprendizaje de los PTIs (Este-2, p. 3)

Por el contrario, en otras empresas el ritmo alto de trabajo es un elemento intrínseco al acompañamiento y un elemento del proceso de cualificación, en la medida que es una de las características propias del mercado ordinario.

Por último, el tamaño, la diversidad de puestos y diferentes tipos de emplazamientos también se identifican como variables que condicionan los procesos de cualificación y acompañamiento. La posibilidad de ofrecer diferentes recorridos formativos (tipo de competencias y niveles de exigencia) es lo que permite ajustarse a diferentes situaciones diseñando 'trajes a medida'. Aquí no podemos establecer una tipología, sino que responde a un continuo de oportunidades, atendiendo a la variable de diversidad de puestos y variedad de emplazamientos.

Hay distintos puestos en la empresa por los que hacen pasar a todo el personal (Centro-2, p. 19)

Sobre una base de perfiles específicos (ayudante de cocinero, ayudante de camarero, y ayudante de servicio) se promueven perfiles polivalentes con posibilidad de ocupar diferentes puestos y/o emplazamientos tanto de forma simultánea como sucesiva (Norte-3, p. 8)

promoviendo en la medida de lo posible la rotación entre puestos para mejorar la formación ofrecida (Centro-1, p.4)

Su itinerario pasa por diferentes puestos de trabajo con variedad de tareas y funciones y en diferentes emplazamientos o centros de trabajo. (Norte-2, p. 10) 


\subsubsection{Recursos humanos: Personas trabajadoras de inserción y personal técnico de acom- pañamiento}

Con relación a las personas trabajadoras de inserción destacan algunos aspectos a considerar. En primer lugar, la forma de acceso a la El es un factor relevante por cuanto influye en el tipo de relación y en el 'contrato psicológico' (Hiltrop, 1996) o vínculo que se establece con cada PTI. En este sentido, el acceso a la El se puede producir desde la propia entidad promotora ( $L a s$ PTI son derivadas por la Fundación -Este-4-) o a través de los servicios sociales o de empleo.

Con relación a las características de las PTI, aunque en general predominan las empresas que se dirigen a una variedad de personas en situación o riesgo de exclusión, hay algunas entidades que se especializan en jóvenes o mujeres, por ejemplo. Es probable que el nivel de homogeneidad de las PTI esté jugando un papel en el grado de estandarización de los procesos de acompañamiento (explícitos o implícitos). Aunque no lo podemos afirmar con rotundidad, hay algunos indicios de que aquellas El que trabajan con colectivos más homogéneos tienden a definir con más claridad algunos procesos, como, por ejemplo, la formación o la transición a la empresa ordinaria. La empresa Norte-3, especializada en jóvenes sería un ejemplo de ello.

Pero más relevante que este aspecto, nos resultan los diferentes niveles de necesidades de apoyo para la mejora de la empleabilidad y los niveles previos de cualificación personal, social y laboral. En este sentido, aunque no es posible diferenciar las empresas en base a esta variable, si se puede establecer una 'clasificación' de las PTI atendiendo a si sus necesidades son bajas, medias 0 altas en la cualificación profesional y lo mismo con relación a su acompañamiento social ('cualificación personal'). En cualquier caso, es relevante destacar que no se han identificado procedimientos o herramientas de evaluación que contribuyan a identificar y objetivar, más allá de la experiencia de cada El, los diferentes perfiles o la intensidad de necesidades de apoyo.

Con relación a los perfiles profesionales y su articulación, en todas las empresas se diferencian los perfiles de Técnico de Acompañamiento y Técnico de Apoyo a la Producción, aunque sus funciones adoptan formas muy diversas, atendiendo sobre todo al mantenimiento de perfiles especializados con clara diferenciación de funciones entre unos y otros 0 , en otros casos, perfiles mixtos

las PTP tienen perfil mixto (técnico y social), característico de la entidad promotora, con una orientación formativa y con funciones también de acompañamiento. (Norte-3, p. 7)

En este sentido, tampoco se aprecia una relación directa con los resultados de las empresas. Aunque la existencia de dos perfiles diferenciados obedece a la lógica de las diferentes dimensiones de las El, su funcionalidad se relaciona con los mecanismos de diálogo y coordinación para que no se conviertan en procesos paralelos, pero con pocas conexiones entre ellos. Por otra parte, los perfiles mixtos son interesantes en el marco de entidades en los que los objetivos sociales están muy presentes en la cultura de las empresas, dándose interesantes sinergias entre las vertientes productiva y formativa de la empresa. 


\subsection{El proceso de acompañamiento}

Como hemos señalado, el acompañamiento como metodología de intervención es el proceso que permite articular los apoyos que precisa la persona tanto con relación a su desempeño laboral como a la mejora de su empleabilidad y que toma como referencia las aspiraciones y metas de las personas. En este sentido, se convierte en el aspecto central que permite proyectar la participación de la persona en la El hacia el objetivo de la inserción laboral. En el análisis de los informes, emergen dos aspectos clave en el proceso de acompañamiento: el grado de estructuración del proceso de acompañamiento y las diferentes fases que lo componen.

\subsubsection{Grado de estructuración del proceso de acompañamiento}

El análisis de los informes revela diferentes prácticas con relación al grado en que el proceso de acompañamiento, como tarea central o "leitmotiv" de las empresas, se estructura o sistematiza.

Las El se mueven entre considerar la estructuración del acompañamiento como eje central de su trabajo, hasta otras que realizan el acompañamiento de un modo más intuitivo, sin soportes de trabajo y guiados por su experiencia. Esto tiene una repercusión en el nivel de explicitación de las propuestas de itinerario personal y formativo de las PTI.

En aquellas El en las que se estructura más el acompañamiento, comienzan por la firma de un compromiso u acuerdo de inserción en el que se contemplan diferentes fases y que varían de unas El a otras:

Cada PTI tiene su acuerdo de inserción realizado en su entrada a la El. Este acuerdo incluye cuatro áreas: socio personal, laboral y profesional, formativa y otros, que se encuentran definidas en el Plan de Trabajo y en el Itinerario de inserción socio laboral. (Este-2, p.16)

Cada PTI tiene su acuerdo de inserción realizado en su entrada a la El. Este acuerdo incluye cinco apartados: situación personal actual, itinerario sociolaboral, puntos fuertes, aspectos a mejorar y plan de trabajo. (Centro-1, p. 15)

En la medida que el acompañamiento implica la formulación de metas compartidas, la percepción que las PTI tienen acerca de su situación y motivos de encontrarse trabajando en la El será la base sobre la que se formularán esas metas. En este sentido, es interesante destacar que la percepción de las PTI es muy diversa. En algunos casos refieren a sus itinerarios personales y en otros remiten a circunstancias sociales, aparentemente ajenas a su propia participación en las mismas.

En este sentido, algunas de las El creen que el mayor grado de estructuración del proceso favorece la toma de conciencia e implicación de la PTI en el proceso: 
Todas (PTI) son conscientes del plan de trabajo que tienen marcado con la PTA, sabiendo cuál es la dirección establecida, los objetivos y los pasos que han de seguir para lograrlos. Asimismo, han sido capaces de comentarnos los reajustes en este plan y explicar a qué se han debido, mostrando una conciencia clara de su situación personal, su situación en la El y de cómo han de trabajar para llevar a cabo las metas propuestas (Centro-2, p. 6).

Lo que también se relaciona con la valoración que las personas hacen del seguimiento de su proceso.

las PTIs no viven las reuniones con tensión, sino que las reconocen como un momento para hablar, explicar cómo se sienten y seguir avanzando en su proceso (Centro-2, p. 15)

el seguimiento no consiste en estar encima de los PTIs, sino mostrarse disponibles y accesibles (Norte-1, p. 15)

\subsubsection{Las fases del acompañamiento}

Como hemos señalado, el proceso de acompañamiento de las PTI se tiende a estructurar en diferentes momentos o fases, sobre las que se recogen datos relevantes en los informes.

Como característica general destaca el hecho de que la intensidad del apoyo y acompañamiento se concentra en una primera fase asociada a la incorporación de la persona a la El y en la fase de transición o salida de la empresa de inserción. Por su parte, el momento intermedio -el más extenso en el tiempo- aparece más diluido sin tantas referencias expresas a cómo se desarrolla.

En el proceso de inserción, parece lógico que en el momento inicial o fase de incorporación vaya asociada a una mayor intensidad en el acompañamiento, en la medida que se produce el aprendizaje de las tareas vinculadas al puesto de trabajo y que se desarrolla el proceso de diagnóstico o identificación de las necesidades de apoyo a nivel social y personal.

Ios aprendizajes se realizan en el propio puesto de trabajo, en gran medida en los primeros meses de desempeño del puesto, con el avance en la El las PTI afianzan otro tipo de aprendizajes (trabajo colaborativo, planificación, proactividad) (Norte-2, p. 15).

Como matiza otra El en la fase inicial, se da mucha importancia a las habilidades sociales siendo la mejora de las habilidades sociales, es también muy relevante y se produce en muchos casos, trabajándose en mayor medida en la acogida e inicios de la fase de seguimiento (Este-4, p. 11).

La fase de transición al mercado laboral ordinario es la otra fase que concentra el esfuerzo del personal técnico de la El. La salida de la empresa y la necesidad de activar y apoyar a la persona en el proceso de búsqueda de empleo, implican una intensificación del acompañamiento 
Se observa una importante dedicación de la empresa al cierre del proceso con objeto de facilitar transiciones exitosas al mercado de trabajo (Este-4, p. 17).

se acerca la finalización del contrato de inserción, la PTA trabaja con cada PTI búsqueda de empleo, utilizando entrevistas de empleo no por el hecho de encontrarlo ya, sino para practicar esas situaciones (Centro2, p. 17)

En ocasiones las El comparten dicho trabajo con la Entidad Promotora, algunas de las cuales tienen esta fase muy estructurada, y el personal de la El se centra en la orientación laboral a la PTI y en el acompañamiento en la búsqueda de empleo.

La orientación profesional propia de la fase de transición hacia un contexto sociolaboral no protegido es considerado un proceso de aprendizaje intencional desarrollado conjuntamente desde (la Entidad Promotora) y la El, en el que el acompañamiento conlleva ir ampliando la autonomía en la búsqueda de empleo: elección del objetivo profesional, elaboración del currículum, generación de un listado de empresas, dirigirse a las empresas, búsqueda por Internet (visitar webs de las ETTs, cumplimentar los formularios, revisar los anuncios de ofertas de empleo...) (Este-4, p. 23).

Por último, señalar que en esta fase como en las anteriores, tanto la intensidad como la duración puede variar y en ocasiones acortarse según las necesidades, condiciones de empleabilidad, oportunidades laborales para la PTI, tal y como se señala siempre: "en beneficio de la propia PTI", (Centro2, p. 18)

De todas formas, llama la atención que la fase más larga, de seguimiento, tenga escasas referencias o descripciones, bien para identificar avances en los procesos de las personas o para identificar situaciones de estancamiento.

\subsection{La formación y cualificación en las Empresas de Inserción}

El análisis de los informes de investigación nos ha permitido identificar algunas claves de los procesos formativos que tienen lugar en las El resultado de la adaptación del modelo de trayectorias de aprendizaje de Eraut $(2009,2007)$ y de Eraut et al. $(2005,2004)$, como hemos explicado en otro lugar para la formación en el puesto de trabajo (Marhuenda, Bernad y Navas, 2010), la participación en procesos de aprendizaje formal y acreditación (Chisvert et al., 2015, 2018; Guillera y Chisvert, 2018), y la conciencia y rol de las PTI (Marhuenda, 2018), las tres subsecciones que desarrollamos a continuación.

Aunque la formación y la cualificación profesional juegan un papel central en la propia misión de las El, su desarrollo práctico es diverso atendiendo al conjunto de variables que identificábamos al hablar de las variables organizativas, así como las del acompañamiento. Por ejemplo, y como hemos 
señalado, el nivel de complejidad de los puestos de trabajo contribuye directamente a la necesidad de desarrollar más o menos competencias. Por otra parte, aquellos puestos de trabajo con referencia en el Catálogo Nacional de Cualificaciones, pueden ir asociados a la obtención de certificados de profesionalidad con el impacto que ello tiene tanto en la estructuración de la formación, como de reconocimiento en el mercado de trabajo. Estos aprendizajes desarrollados en el propio puesto de trabajo pueden tener componentes tanto formales como informales.

Al mismo tiempo, hay otro conjunto de competencias -sobre todo las personales y sociales- que se espera que tengan un impacto, más allá de los puestos de trabajo, en otras dimensiones de la vida de las personas.

Por otra parte, la mejora de la empleabilidad que permite acceder a puestos de trabajo en empresas ordinarias puede implicar procesos formales de aprendizaje que han de buscarse en paralelo a la El, aunque pueden formar parte del proceso de acompañamiento y cualificación que se facilita desde la empresa.

En este apartado mostramos los procesos formativos y de aprendizaje que se producen en las El, ya sean fruto de aprendizaje informal o bien de prácticas formalizadas.

\subsubsection{La formación en el puesto de trabajo}

A pesar de que todas las El cumplen con los requisitos administrativos establecidos en la normativa con relación al acompañamiento y la cualificación (contrato, plan individualizado, seguimiento de las fases del proceso), hemos encontrado pocas evidencias de su uso sistemático como soporte para los procesos formativos. Tanto el plan personal de inserción como, sobre todo, su seguimiento se desarrollan mayoritariamente mediante procesos informales; los acuerdos formales están orientados más al largo plazo y no a las decisiones a corto plazo en el trabajo de acompañamiento.

un aspecto que hay que señalar es el escaso soporte documental que hemos observado tanto en la relación con las personas como en el trabajo entre profesionales. Buena parte de los planes personales a los que hemos accedido son estándar y parece que tiene una función más administrativa que como herramientas de soporte al trabajo formativo (Norte-3, p. 19).

Con frecuencia, en muchas empresas los procesos de formativos son subsidiarios a los procesos productivos, y así es percibido por las tres partes implicadas. En este sentido, el aprendizaje

se espera sea el resultado situacional de compartir el espacio y el trabajo. La formación es el resultado del propio desempeño -acompañado en las situaciones problemáticas- en el trabajo real (Este-4, p. 16).

Más allá de los planes formales de trabajo, hemos encontrado ejemplos en los que la exigencia y calidad del aprendizaje está estrechamente vinculada a la definición de perfiles profesionales. Esto 
se aprecia con claridad en las ocupaciones de restauración y lavandería, también en alguna de las de reciclado (éste es el caso en cuatro empresas: Centro-1 y Centro-2, Norte-3 y Este-4). En la mayor parte de estos casos la formación previa en el sector desempeña un papel en el proceso de selección y continúa, tomando como referencia las competencias profesionales propias del puesto, en el desempeño del propio puesto de trabajo.

En resumen, dos son las cuestiones a las que nos remite el aprendizaje en el puesto de trabajo. Por una parte, la 'calidad formativa del puesto', vinculada a variedad, complejidad y especialización de las tareas que implica. Por otra, los apoyos a la formación relacionada con la cualificación del personal de apoyo y con la organización de los procesos de trabajo. A continuación, nos detenemos en ambas.

\section{Potencial formativo del puesto de trabajo}

Aunque la mayor parte de los puestos de trabajo en las El son de baja cualificación (nivel 1, ocasionalmente nivel 2), hemos identificado varias dimensiones vinculadas al puesto de trabajo en las que la progresión de la PTI era posible desde su incorporación inicial a la empresa. En este sentido, los puestos de trabajo oscilan entre dos situaciones o polos. Por una parte, puestos de trabajo que implican variedad y complejidad de competencias y, por otra, puestos de trabajo rutinarios y que demandan de la persona que los ocupa un conjunto limitado de competencias que además son sencillas de realizar. En el primer caso se encuentran los puestos de empresas de hostelería, limpieza, lavandería o jardinería, en los que la polivalencia suele estar presente, implican habilidades complejas como trabajo en equipo o atención a clientes

Sobre una base de perfiles específicos (ayudante de cocinero, ayudante de camarero, y ayudante de servicio) se promueven perfiles polivalentes con posibilidad de ocupar diferentes puestos y/o emplazamientos tanto de forma simultánea como sucesiva. La referencia de los niveles de cualificación y su relación con el mercado de trabajo hace que haya niveles altos de correspondencia entre los puestos de la El y sus equivalentes en el mercado ordinario aunque con énfasis, como comentábamos, en la polivalencia (Norte 3, p. 8).

En el otro polo nos encontramos con empresas de recuperación, con puestos de trabajo en los que las tareas son rutinarias o están mecanizadas (clasificación de ropa o residuos, por ejemplo), en los que las tareas se aprenden en poco tiempo y las posibilidades de mejora en el desempeño pasan por aumentar la precisión o velocidad de las tareas, dando lugar a poco aprendizaje técnico. Son puestos que no requieren formación previa y, en caso de requerirla, esta se adquiere en un periodo muy breve de tiempo. De todas formas, caben algunas matizaciones en dos direcciones. Por una parte, hemos encontrado, en el sector de la recuperación, puestos de trabajo que dan más oportunidades de aprendizaje en la medida en que las competencias implicadas en su realización son variadas y complejas. 
En algunos casos los propios puestos de trabajo implican cada día cierta cantidad de retos prácticos (montaje y desmontaje de muebles, estibación de la furgoneta, exposición,...) y especialmente los vinculados con la relación con la clientela, que implica un proceso de aprendizaje permanente $y$ asi es identificado por varios PTIs (Norte-1, p. 12)

Por otra, también hemos encontrado estrategias orientadas a 'enriquecer' el desempeño profesional a través de la explicitación y desarrollo de competencias transversales o de la formulación de 'retos productivos':

También se desarrollan las competencias socio-emocionales que hacen referencia tanto a la conciencia emocional que una persona tiene como a la autogestión, la empatía y la gestión de las relaciones. La relevancia de las competencias socio-emocionales se hace patente en distintos ámbitos: procesos de aprendizaje, relaciones interpersonales, resolución de problemas. Junto a ello, también tienen el objetivo de aumentar el bienestar personal y social de las mujeres (Este-1, p. 21).

Las PTI son conscientes del feedback que reciben pues al inicio de la semana el PTP, en lo relativo a clasificación, comparte resultados individualizados y colectivos de producción, establecen records para motivar y asï toman conciencia del objetivo común. PTP ha ideado un sistema público para informar de la producción de cada trabajador y se reúne con cada una para informar de la progresión (Este-2, p. 12).

Estas estrategias pueden contribuir a que las PTI no se sientan estancadas y perciban que están aprendiendo y mejorando su desempeño profesional.

\section{Calidad de los apoyos a la formación en el puesto de trabajo}

Como hemos señalado, los apoyos que reciben las personas en el puesto de trabajo son claves en los procesos de aprendizaje. Estos apoyos pueden provenir del personal técnico, pero también se pueden referir al apoyo de otras personas 0 de los recursos y procedimientos disponibles en el puesto. Hemos podido identificar en varias de las empresas los siguientes apoyos vinculados al aprendizaje en el puesto de trabajo:

Preguntar a quien sabe. Identificar a las personas con recursos y saber acudir a ellas en el momento necesario, es un aprendizaje que tiene lugar en el seno de la organización y que a su vez es la llave a otros aprendizajes.

La comunicación fluida es uno de los aspectos que fundamentan esta adquisición de autonomía: preguntan, observan para aprender y para poder hacer ellas después la tarea determinada (Centro-1, p. 8)

Hay mucho tiempo disponible para la consulta en la ocupación de recogida y no supone ninguna pérdida de tiempo en producción al producirse en mayor medida durante los desplazamientos. A partir de la segunda fase del itinerario las consultas son informativas (consultas sobre dónde se dirigen en primer lugar, o sobre dónde van a almorzar) y la formación 
se mantiene en ámbitos relativos a competencias transversales (taller sobre las emociones, reuniones con la PTA) (Este-4, p. 19)

Como hemos destacado las PTI preguntan (consultan) siempre que lo necesitan para obtener información, aunque la primera opción es la búsqueda de soluciones individuales. Saben identificar a las personas con recursos, principalmente en las figuras de PTA/PTP, PTP para el trabajo y PTA para cuestiones personales. Se ha observado que las PTI son conscientes de que reciben feedback, se les recapitulan los errores o se les hace reflexionar sobre 10 acontecido (Norte-2, p. 17)

Apoyo entre compañeros, con y sin experiencia laboral, con distinta trayectoria dentro de la empresa, organización de equipos de trabajo. Incorporado a equipos de trabajo, este apoyo va más allá del hecho de trabajar conjuntamente y pasa por encontrar personas de referencia, en las que poder confiar, a las que poder tomar como ejemplo o bien con las que poder contar para no tener como único interlocutor válido a PTP o PTA.

En ocasiones este aprendizaje (sobre todo en el momento inicial) se desarrolla junto a otras PTI veteranos (...) Todos ellos tienen claro que en su fase inicial en la El fueron ayudados y acompañados por otros trabajadores más veteranos y que a ellos les tocará hacer lo mismo (Norte-1, p. 12)

Enseñanza directa en el puesto que consiste: en instrucciones previas a la realización del trabajo, en proporcionar ejemplos de cómo realizar determinadas tareas (ergonomía, planificación, secuencia de tareas, protocolo de pasos), dar indicaciones para la observación, ejercer de modelo, reflexionar sobre el trabajo realizado, invitar a la reflexión propia o bien echar mano de manuales de instrucciones o consejos apropiados.

El desempeño del trabajo es el eje en torno al cual tienen lugar los aprendizajes en esta empresa, tanto los estrictamente vinculados al trabajo como los que afectan al desarrollo personal y social: la constitución de la propia identidad tiene en el trabajo, en el trabajo digno, un componente importante, como también lo tiene el respeto hacia las otras personas y la confianza en el desempeño de su trabajo de forma efectiva (Centro-2, p. 15)

Los procesos de aprendizaje se realizan al "calor" de la tarea. Es el desempeño real el que lleva al aprendizaje. En ocasiones este aprendizaje (sobre todo en el momento inicial) se desarrolla junto a otros PTIs veteranos. Siendo el papel de PTP más puntual y de orientación ante dificultades más concretas. El aprendizaje es el resultado de la propia práctica, acompañada en ocasiones de manera reflexiva por PTP (Norte-1, p. 12)

Trabajo en equipo, consistente en organizar la producción en equipos de trabajo en los que se consigan relaciones humanas satisfactorias además de eficaces. Las decisiones sobre los equipos, su composición, los encargos que se les hacen, la estabilidad o su transitoriedad, se toman con frecuencia atendiendo no sólo a necesidades productivas, sino también a la progresión en los itinerarios de inserción. Pero, más allá de los equipos de trabajo, hemos encontrado también actividades formativas con carácter grupal, lo que facilita la formalización de algunos tipos de aprendizaje. 
Destacamos, ante todo, el trabajo en equipo. El funcionamiento por turnos y la coordinación necesaria conlleva el aprendizaje de la comunicación entre diferentes personas y del control de emociones. Estos aspectos se reconocen además como aprendizajes muy positivos por parte de las PTI (Centro-1, p. 11).

En algunas empresas hay ejemplos de actividades grupales orientadas a aprendizajes, tanto de carácter técnico como personal o sociolaboral

En la El se hacen actividades en grupo como reuniones grupales de información de criterios de clasificación (se ha observado una donde las trabajadoras participan de forma natural planteando dudas y opiniones) que se realizan siempre según necesidad y volumen de trabajo. Son momentos de aprendizaje no solo de tareas concretas de trabajo, sino también de relaciones personales (Este-2, p. 11)

El funcionamiento por turnos y la coordinación necesaria conlleva el aprendizaje de la comunicación entre diferentes personas y del control de emociones. Estos aspectos se reconocen además como aprendizajes muy positivos por parte de las PTI (Centro-1, p. 11)

Por último y con relación a la Sistematización de los procesos de trabajo, aunque es probable que de manera informal estén presentes, sólo los hemos encontrados en aquellas empresas que utilizan el trabajo en cadena, donde se tienen que establecer con claridad las secuencias y ritmo de trabajo

La sistematización y organización del trabajo en cadena conlleva el tomar conciencia de que cada tarea individual forma parte del resultado colectivo y que el éxito del trabajo de la PTI es el éxito común en cuanto a logros. La organización por turnos requiere de coordinación y comunicación, de informar y saber qué ha hecho el turno previo y qué hay que preparar para el siguiente turno, algo que las PTI asumen como parte de sus tareas (Centro 3, p. 9).

\subsubsection{Participación en procesos de aprendizaje formal y acreditación}

La participación en procesos formativos formales, que puedan incluir la acreditación o titulación, es un aspecto que mejora la empleabilidad. Conviene resaltar que una parte importante de las personas trabajadoras de inserción han estado excluidas de ese tipo de procesos formativos. Además, compaginar procesos de trabajo con procesos formativos implica niveles de motivación y circunstancias vitales que lo hagan posible.

En varias empresas estar incorporado a un proceso formativo es requisito para estar ocupando un puesto de trabajo

En el momento de la observación las PTI, salvo quienes ya disponen de un grado medio, están siguiendo un plan complementario de formación académica, en unos casos para la consecución del título de Graduado en Secundaria Obligatoria y en otros de un Grado Medio de Formación Profesional (Norte-3, p. 8) 
Prácticamente todas tienen como objetivos la formación fuera de la El para la obtención del GESO o, en un caso, del Bachillerato. Estos objetivos no se han alcanzado en el período que han abarcado las visitas, aunque sí se han trabajado por parte de algunas PTI que van a organizaciones educativas en su tiempo libre (Centro-1, p. 12).

En otras, donde la formación es una prioridad de la empresa, se enfatiza la obtención de certificados de profesionalidad

Se han homologado aulas en la Entidad promotora para la impartición de certificados de profesionalidad de mozo de almacén. Las acciones formativas formales (cursos, seminarios,...) o la participación en acciones formativas externas, se llevan a término moderadamente. Se hace explícito el inicio del proceso encaminado a la acreditación de competencias (Este-4, p. 5).

También se puede destacar alguna empresa que realiza acciones formativas en la propia empresa como complemento a la formación en el puesto de trabajo

También los viernes se organizan sesiones de formación en la empresa con contenidos variados: estilos de comunicación verbal y no verbal, trámites de IRPF y otros, prevención de riesgos laborales; aprovechando que ese día se pueden solapar turnos porque la carga de trabajo es algo más ligera (Centro-2, p. 17)

De todas formas, también se ha detectado que, en varias de las empresas estudiadas, la formación es prácticamente irrelevante y apenas se reseñan procesos formativos formales.

\subsubsection{Conciencia y rol de las PTI: Trabajar más que aprender}

Como hemos señalado al hablar de los procesos de acompañamiento, la percepción y conciencia de las PTI sobre su participación en la El es un aspecto crucial. En este sentido, es muy relevante destacar que las PTI se perciben, por encima de todo, como personas trabajadoras. Para alguien que ha estado en situación de vulnerabilidad, el hecho de trabajar contribuye a una imagen positiva y normalizada de sí misma.

De todas formas, la mayoría de las PTI también tiene conciencia de estar en un proceso de cualificación y mejora de su empleabilidad

Todas son conscientes del plan de trabajo que tienen marcado, sabiendo cuál es la dirección establecida, los objetivos y los pasos que han de seguir para lograrlos. Asimismo, han sido capaces de comentarnos los reajustes en este plan y explicar a qué se han debido, mostrando una conciencia clara de su situación personal, su situación en la El y de cómo han de trabajar para llevar a cabo las metas propuestas. La capacidad de reflexionar sobre la situación personal es destacada como uno de los aprendizajes más relevantes que realizan en la El (Centro-1, p. 6) 
La conciencia que tienen las PTIs de la necesidad de seguir aprendiendo y mejorando, así como la capacidad de reflexionar sobre sus puntos fuertes y débiles. Incluso aquellas que muestran un dominio de las tareas insisten en que siempre se puede mejorar el desempeño en los diferentes puestos de la El. (Este 3, p. 3)

A pesar de ello, también se detectan, sobre todo en algunas empresas en las que la dimensión formativa está menos presente, algunas contradicciones en este doble papel que tienen las personas en las El. En estas ocasiones el hecho de disponer de un empleo puede contribuir a diluir otras necesidades de recualificación o de mejora en su situación personal y social

La tarea productiva y sus resultados cobran mucho peso y esto se traslada a los trabajadores. Estos tienen una conciencia relativa a cerca de su situación, matizando su situación como de inserción "laboral" y no "social". En este sentido para ellos la El es sobre todo una oportunidad laboral, con cierto "nivel" salarial, frente a las oportunidades que encuentran en el mercado libre (Norte 2, p. 17).

En cierto modo, la seguridad que concede la condición de trabajador puede pasar por encima de otra condición, la de inserción, que puede quedar en segundo plano. Saberse contratado, recibir evaluaciones positivas del desempeño, puede dar lugar a que la PTI se sienta reforzada en su hacer y su saber que, junto a la baja cualificación que en ocasiones puede demandar el puesto, da lugar a olvidarse de la condición de inserción, de la temporalidad del contrato de inserción, de las dificultades y obstáculos a los que deberá hacer frente en el mercado de trabajo ordinario.

También es relevante destacar que este equilibrio entre los dos roles que desempeñan las PTI está más presente en las primeras fases del proceso, mientras que, en la fase de transición y la proximidad del momento de abandonar la El para ir al mercado de trabajo ordinario, emergen con claridad las contradicciones.

\section{Discusión de resultados: agenda de temas}

A continuación, se discuten los principales resultados y se avanzan algunas implicaciones tanto para las propias El como para la investigación, atendiendo a los tres niveles de análisis que se han utilizado en el estudio. 


\subsection{Con relación al marco organizativo de las empresas}

1.Diversidad de las El y necesidad de enfoques multidimensionales en el análisis de las prácticas de acompañamiento y cualificación

Como se reconoce en la literatura y los resultados de esta investigación, los datos de este trabajo vuelven a resaltar que las El se configuran como realidades complejas y heterogéneas cuyo funcionamiento se ve influido por factores diversos, tanto macro, meso como micro. Las prácticas de acompañamiento y cualificación no se entienden, ni se pueden mejorar sin atender a ese conjunto de factores. En ese sentido, es necesario disponer de esquemas y herramientas de análisis globales, que pongan el énfasis en las interacciones y en las influencias mutuas entre dimensiones y factores.

En este sentido, identificamos cuatro niveles interconectados de análisis e intervención:

\section{Figura 1. Niveles de análisis de las EI}

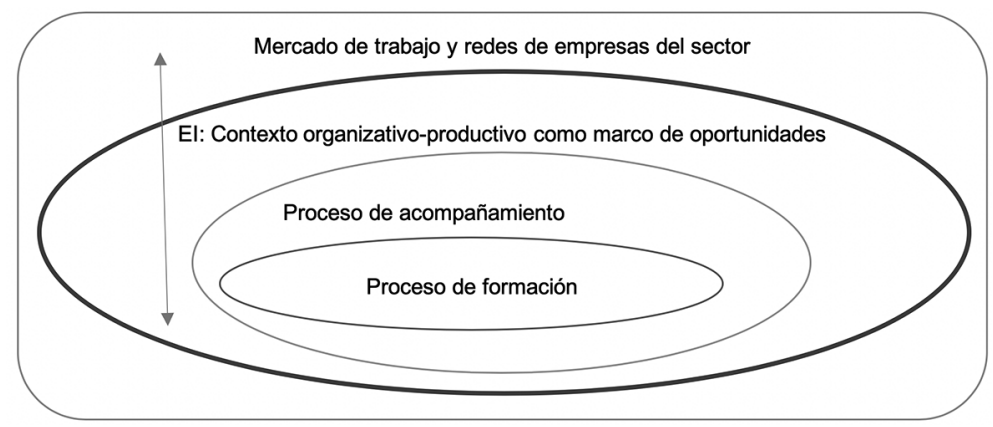

2. Las redes de colaboración como condicionantes clave de los procesos de acompañamiento y cualificación

Como se ha podido comprobar y como también se señala en la literatura (Adam et al., 2017), los factores que inciden en cómo se desarrollan los procesos de apoyo y cualificación son múltiples atendiendo a las diferentes dimensiones de funcionamiento de las empresas sociales. A pesar de que en esta investigación no se han analizado todas sus dimensiones, sí se han identificado algunos de estos factores que condicionan la forma en que se desarrollan dichos procesos. En particular, aparece como condicionante el grado e intensidad de las relaciones que mantiene la empresa con las entidades promotoras, las empresas ordinarias y con los recursos formativos. En este sentido, más que existir unas características que aseguren el éxito, parece que podemos hablar de patrones de interacción complejos y dinámicos, en los que se deben desarrollar mecanismos que aseguren el equilibrio entre 
los diferentes componentes y niveles. Así, prestar atención a los mecanismos de colaboración y coordinación tanto con la entidad promotora como con los recursos formativos, el entorno productivo y las empresas ordinarias se convierte en una cuestión estratégica.

3. Las empresas de inserción como estructuras transicionales: La importancia de las relaciones y la vinculación con el mercado de trabajo

Tanto para el propio desarrollo de los procesos productivos como para facilitar los procesos de incorporación de los PTI a las empresas ordinarias es preciso que las El hagan un trabajo de ubicación en el mercado de trabajo ordinario en el que van a participar con propuestas de "inserción" de ellas mismas como empresas conectadas con el entorno. Este proceso puede ser facilitado a través de la creación de estructuras y canales de colaboración, así como por la presencia de perfiles profesionales relacionados con la prospección laboral, la intermediación o de empleo con apoyo. De todas formas, hay que tener en cuenta que este tipo de estrategias serán diferentes según los sectores productivos en los que se mueven las empresas, atendiendo al grado de ajuste entre los perfiles profesionales de la empresa y sus equivalentes en el entorno.

4. La organización productiva como matriz para el diseño de los procesos de acompañamiento y cualificación

La toma de decisiones acerca de cómo se organizan los procesos productivos en las El se ve influenciada por numerosas variables que toman como referencia la necesidad de ser proyectos económicamente viables. Ahora bien, para poder cumplir con sus objetivos sociales, y particularmente, su capacidad formadora, es preciso que se incluyan en esa toma de decisiones criterios de carácter social y pedagógico. En cualquier caso, y aceptando que los procesos productivos tienen una dinámica propia es preciso desarrollar estrategias y procesos que complementen la diversa, según empresas, potencialidad cualificadora de su dinámica productiva. Esto implica que es probable que no se puedan estandarizar para todas las empresas los procesos formativos, aunque será imprescindible que cada empresa construya su propio diseño formativo a partir del análisis de las potencialidades y limitaciones que su actividad productiva presenta. El tamaño, la diversidad de puestos y diferentes tipos de emplazamientos también se identifica como una variable que condiciona los procesos de cualificación y acompañamiento. La posibilidad de ofrecer diferentes recorridos formativos (tipo de competencias y niveles de exigencia) es lo que permite a cada empresa ajustarse a diferentes situaciones de sus PTI diseñando 'trajes a medida', a la vez que se corre el riesgo de no diseñar ese traje.

5. Las principales variables que inciden en la potencialidad formadora de la El son la existencia de una cualificación de referencia en el Catálogo Nacional de Cualificaciones Profesionales, el desarrollo de procesos de trabajo colaborativo y en equipo/grupo, los niveles de exigencia productiva (aunque con una función facilitadora u obstaculizadora, según los casos) y la diversidad de puestos y espacios de trabajo. 
Partiendo del hecho de que cualquier desempeño en un contexto laboral implica algún tipo de aprendizaje profesional, el problema se sitúa en el tipo, calidad e impacto de ese aprendizaje. Por tanto, un primer elemento que identificamos se relaciona con el grado, calidad o tipo de las experiencias formativas que la El promueve en el mismo puesto de trabajo. En este sentido, cabría diferenciar entre lo que el propio puesto de trabajo da de sí (lo que se puede aprender en él) y lo que la empresa incorpora para enriquecer el proceso de inserción (lo que se propone enseñar a la PTI y cuya responsabilidad recae en el personal técnico de acompañamiento).

Respecto al propio puesto de trabajo hay que tener en cuenta que, en la medida en que hay puestos cualificados (en cocina, en jardinería, en servicio de bar o ayuda a domicilio) se requiere una formación previa para acceder al puesto de trabajo, que en ocasiones ha sido proporcionada por la entidad promotora y, en otras, es un conocimiento previo que la propia PTI aporta en el proceso de selección. Sin embargo, en los puestos de trabajo de baja o muy baja cualificación (una parte del sector de recuperación) no es necesaria formación previa, aunque alguno de ellos (lavandería industrial, por ejemplo) sí que proporcione la posibilidad de aspirar a un reconocimiento de competencias, puesto que hay una progresión clara tanto de dificultad y complejidad de las tareas como de velocidad y precisión en su desempeño.

Por último, la obtención de dicho certificado o cualificación profesional debiera ser uno de los hitos que identificaran el inicio de la fase de transición al mercado de trabajo ordinario, puesto que disponer del mismo equipa a las PTI para competir por un puesto de trabajo en condiciones apropiadas, mientras que no hacerlo, allí donde sí sería posible, supone enviarlas al marcado de trabajo en una posición de debilidad.

\subsection{Con relación a los procesos de acompañamiento}

6. Más allá de un lenguaje y referencias compartidas (FAEDEI \& AERESS, 2014), los procesos de acompañamiento se desarrollan con niveles muy diferentes de sistematización y explicitación.

La diversidad de formas en las que se desarrollan los procesos de acompañamiento (objetivos, fases, herramientas, estrategias...) sugiere que otras variables o condicionantes (la cultura organizativa, los procesos productivos o las características de los profesionales o trabajadores) tienen más influencia que las propias orientaciones teóricas acerca de cómo se desarrollan los procesos de acompañamiento. Más allá de esta diversidad, es posible identificar las fases de acogida y diagnóstico de la empleabilidad y la fase de búsqueda de empleo como los momentos clave del proceso de acompañamiento.

7. Necesidad de desarrollar un sistema fiable y válido de evaluación de la empleabilidad que contribuya a estandarizar el proceso de selección, así como la evaluación e impacto de las El. 
Una contribución relevante a la necesidad identificada de articular el proceso de acompañamiento se puede vincular a la necesidad de sistematizar los procesos de evaluación de necesidades e identificación de apoyos que permitiera facilitar los procesos de selección, pero también estimar el impacto de las diferentes El en la mejora de la empleabilidad, más allá de las tasas de inserción. Al mismo tiempo, desarrollar un sistema de seguimiento hasta 3 años después de abandonar la empresa de inserción podría contribuir a evaluar el impacto de las El a medio y largo plazo.

8. La fase inicial y fase de salida aparecen como los momentos clave del acompañamiento.

La fase inicial tiene gran potencialidad porque permite acceder a un empleo a través de un contrato con garantías y contribuye a que las personas puedan sentirse competentes. Además, es un momento de adaptación, clarificación y definición del itinerario personal en el marco del contrato laboral. La fase de salida o transición es el otro momento clave, aunque también es un momento de crisis al manifestarse una tensión entre el rol de trabajador y el rol de persona en proceso de inserción.

9. En estas fases, aunque particularmente en la inicial, la elaboración que las personas hacen de su situación y rol, y su explicitación en planes personales, emergen como el eje de la intervención profesional.

En este sentido, la estructuración y sistematización del proceso de acompañamiento y de formación parece que es un factor que contribuye al desarrollo de una mayor conciencia de la situación por parte de las personas y a una progresiva autonomía en su proceso de inclusión laboral y social.

\subsection{Los procesos formativos en las El}

10. El análisis y mejora de la oferta formativa de las El tiene que considerar el conjunto de condicionantes estructurales, organizativos y metodológicos

En este sentido, hay que resaltar que los procesos formativos, en primer lugar, están fuertemente condicionados por la variedad y complejidad de las competencias implicadas en los diferentes puestos de trabajo y su relación con los niveles de cualificación de cada puesto de trabajo. Las condiciones del puesto y las necesidades y conciencia de la PTI son los dos factores que afectan a la relevancia de lo personal o lo profesional en cada fase.

11. El análisis de los procesos de formación en la El tiene que considerar la 'calidad formativa del puesto' y la calidad y variedad de los apoyos que la empresa presta y que supone avanzar en la sistematización de los aprendizajes (informales) en el puesto. 
12. Necesidad de diseñar un modelo formativo propia de las El que, tomando como referencia la formación en el puesto, desarrolle una variedad de estrategias para asegurar una cualificación con encaje en el mercado ordinario de trabajo

Reconociendo que la propia participación de las personas en las El y el desempeño de un puesto de trabajo implican siempre, en grados diversos, aprendizajes profesionales de carácter no formal, a la luz de las limitaciones identificadas en el desarrollo de los procesos formativos, resulta necesario avanzar en el diseño de un modelo formativo propio que promueva la formación en el puesto de trabajo y que la complemente con otras estrategias, espacios e instituciones que aseguren la cualificación para la incorporación al mercado ordinario.

13. El modelo formativo propio tiene que incluir dos referencias clave: perfiles profesionales asociados a los puestos de trabajo y la sistematización de los apoyos a través de rutinas formativas asociadas al desempeño profesional

Muchos de los procesos de aprendizaje en el puesto de trabajo son de carácter informal, incidental o tácito (Eraut, 2000; Garrick, 1998; Marsick \& Watkins, 2001), y la adquisición de conocimiento, competencias 0 saber se produce como efecto colateral de los procesos productivos (Eraut, 2004). Sin embargo, en las El ese efecto no sólo es colateral, sino que forma parte del objeto mismo del contrato, que conlleva el desarrollo de un itinerario de inserción.

En este sentido, puede ser interesante explicitar las condiciones mínimas que se deben dar para que ese aprendizaje informal produzca una mejora de la empleabilidad. Eso tiene que ver con la creación de espacios, tiempos, agrupamientos y roles (elementos estructurales) que se pueden crear para que desde la organización se optimicen, en una determinada dirección, los procesos de aprendizaje que de hecho se producen fruto de la participación de las personas en un proceso productivo, en ocasiones con un fuerte carácter rutinario.

Parece oportuno, por lo tanto, insistir en una sistematización de los procesos informales de aprendizaje. Los elementos que van a hacer que esos procesos se mejoren son, por este orden, seis pasos:

1. Empoderamiento de la persona a través de enfatizar su participación tanto en los procesos productivos como en su propio itinerario.

2. Aumento de la conciencia de estar aprendiendo y de saberse en proceso de inserción.

3. Sistematización y control de los elementos clave del proceso productivo.

4. Aumento de las interacciones con técnicos y compañeros como oportunidades de aprendizaje a diferentes niveles (Marsick \& Watkins, 2001), enfatizando la colaboración y el trabajo en equipo, al servicio tanto del proyecto de la empresa como del propio itinerario de inserción. 
5. Aprovechar las incidencias o incidentes como oportunidades singulares de aprendizaje, estableciendo como secuencia básica de aprendizaje: identificar problemas y/o retos, buscar soluciones, valorar su eficacia.

6. Diseñar espacios y momentos de revisión y evaluación grupales e individuales del trabajo realizado e identificación de las mejoras a incorporar (Bernad, Navas y Marhuenda, 2010).

Se trata, en definitiva, de crear una cultura y apoyo para el aprendizaje en las organizaciones, capaz de establecer nexos entre las relaciones a nivel individual, grupal y organizacional.

14. En el diseño del modelo formativo será necesario completar los procesos de formación en el puesto con la participación en procesos de cualificación y acreditación de carácter formal, que complemente la formación en el puesto de trabajo.

Resulta razonable facilitar, en el marco del itinerario, una formación complementaria, que se pueda cursar al margen del trabajo en la empresa, y que contribuya a mejorar la empleabilidad de las PTI. En este sentido las El deben promover un aprendizaje formal y, en lo posible, una acreditación de algún tipo de saber, académico o profesional, con reconocimiento a través de medidas de conciliación, adaptación o mediación con los recursos o programas formativos existentes en el entorno que, sin ser de la empresa, bien pueden contribuir a reforzar elementos del itinerario.

\section{Conclusiones}

En este artículo hemos descrito las prácticas de acompañamiento y formativas de las El. A partir de adoptar un enfoque multidimensional, se han presentado diferentes factores organizacionales que influyen en dichas prácticas. En particular, las relaciones que mantiene la El con las entidades promotoras y, sobre todo, con el mercado de trabajo ordinario y la forma de organización de las actividades productivas. Con relación a los procesos de acompañamiento se han identificado el grado de estructuración y las fases del acompañamiento como las variables más relevantes a considerar. Por último, y con relación a los procesos formativos, se han presentado las prácticas relacionadas con la formación en el puesto, con la participación en procesos formativos formales y su relación con la conciencia y rol de las personas.

En base a estos hallazgos, se ha presentado una agenda de temas que las El tendrán que afrontar en los próximos tiempos y que se pueden resumir en: 
1. Avanzar en la comprensión multidimensional de las El de forma que tanto las prácticas como la investigación aborden de forma holística e integrada los retos a los que se enfrentan. En este sentido, cada El tendrá que considerar la interdependencia entre las tres dimensiones analizadas para, en el marco de su contexto socioeconómico, definir y concretar su oferta.

2. Debido al carácter multidimensional, híbrido y transicional de las $\mathrm{El}$, es necesario avanzar en el desarrollo de estructuras de colaboración y cooperación, tanto internamente como con relación al entorno, que permitan mantener el equilibrio entre los diversos objetivos y dimensiones de las empresas.

3. Esta perspectiva multidimensional tiene que concretarse en el diseño de un modelo pedagógico propio que, partiendo de las oportunidades que ofrecen las diversas actividades productivas, tome como referencia el desarrollo de perfiles profesionales e integre la formación en el puesto -con sistematización de los apoyos a través de rutinas productivo-formativas-, la formación y acreditación de carácter formal y que preste especial atención a las formas en que las personas trabajadoras de inserción perciben su rol.

4. Avanzar en la estructuración de un proceso de acompañamiento que tome como punto de partida la percepción y expectativas de las PTI y que explicite con claridad metas en términos de empleabilidad que se esperan conseguir. La construcción de una herramienta de evaluación de la empleabilidad puede contribuir a ello.

En síntesis, en este artículo se ha presentado un modelo comprensivo de los procesos de gestión, acompañamiento y formación de las El que, considerando su complejidad y diversidad, permite articular y relacionar sus diferentes dimensiones, como la base necesaria para proyectarse en un futuro cada vez más exigente.

\section{Referencias}

ÁLVAREZ, L., CÓZAR, S., MARTíNEZ, E. Y MELÍAN, S. (2019): “Tejiendo alianzas público-privadas para la generación de empleo de inserción", Noticias de la Economía Pública, Social y Cooperativa - Noticias del CIDEC, 60, 55-68.

ANDRÉU, J. (2002): Las técnicas de análisis de contenido: una revisión actualizada, Sevilla, Fundación Centro de Estudios Andaluces.

ABIÉTAR, M., ROS-GARRIDO, A. \& MARHUENDA, F. (2018): "Profesionales de apoyo a la inserción: formación y acompañamiento en empresas de inserción", CIRIEC-España, Revista de Economía Pública, Social y Cooperativa, 94, 155-183. DOI: 10.7203/CIRIEC-E.94.12698. 
ADAM, S.R., AVILES, G., SCHMITZ, D., LUCCHINI, A., FERRARI, D., CAVEDON, E., ... AMSTUTZ, J. (2017): "The WISE's success factors from a multidimensional and multi-stakeholder perspective. An explorative study in the Swiss context", 6th EMES International Research Conference on Social Enterprise, https://irf.fhnw.ch/bitstream/handle/11654/26076/Avil_s_et_al_Switzerland_6EMESFullPaper.pdf?sequence=1.

ASKUNZE, C. (2016): "Empresas de inserción en la economía social. Herramientas para la inclusión sociolaboral", CIRIEC-España, Revista Jurídica de Economía Social y Cooperativa, 29, 1-32.

ASSOCIAÇÃO A3S. (2014): The marketing and coaching functions of work integrated social enterprises (WISE). An exploratory study in 5 European countries, http://www.evtnetwork.it/wp-content/ uploads/2016/11/IO-1_Exploratory-study_Final-Version.pdf.

AUDENAERT, M., VAN DER HEIJDEN, B., CONWAY, N., CRUCKE, S. \& DECRAMER, A. (2019): "Vulnerable Workers' Employability Competences: The Role of Establishing Clear Expectations, Developmental Inducements, and Social Organizational Goals", Journal of Business Ethics, 1-15. DOI: 10.1007/s10551-019-04140-9.

BATTILANA, J., SCHOOL, H.B., SENGUL, M., COLLEGE, B. \& PACHE, A.C. (2015): "Harnessing Productive Tensions in Hybrid Organizations: The Case of Work Integration Social Enterprises", Academy of Management Journal, 58 (6), 1658-1685. DOI: 10.5465/amj.2013.0903.

BEL, P., LEJARRIAGA, G. \& MARHUENDA, F. (2018): "Educación y formación en economía social y cooperativismo", CIRIEC-España, Revista de Economía Pública, Social y Cooperativa, 94, 5-9.

BELASSIR, A., CALABOZO, L. \& TREGUER, G. (2019): "Las empresas de inserción en España", Noticias de la Economía Pública, Social y Cooperativa - Noticias del CIDEC, 60, 39-44.

BLOOM, D. (2010): Transitional Jobs: Background, Program Models, and Evaluation Evidence, Washington, DC: US Department of Health and Human Services, www.acf.hhs.gov/sites/default/files/ opre/tj_09_paper_embed.pdf.

CAMPI S., DEFOURNY J. \& GRÉGOIRE O. (2006): "Work integration social enterprises: are they multiple-goal and multistakeholder organizations?". In: Nyssens M. (ed.), Social Enterprise: At the Crossroads of Market, Public Policies and Civil Society, London: Routledge, 27-49.

CEBOLLA, I., CHISVERT-TARAZONA, M.J. \& GUILLERA, S. (2019): "Reconocimiento y acreditación de las competencias profesionales en las empresas de inserción", Noticias de la Economía Pública, Social y Cooperativa - Noticias del CIDEC, 60, 50-54.

CHISVERT-TARAZONA, M.J., ROS-GARRIDO, A., CORDOBÁ-IÑESTA, A. y MARHUENDA-FLUIXÁ, F. (2015): "Mapa de cualificaciones profesionales acreditables en las empresas de inserción", Certiuni Journal, 1, 36-50. 
CHISVERT-TARAZONA, M.J., PALOMARES-MONTERO, D., HERNAIZ-AGREDA, N. \& SALINAS, M. (2018): "Trayectorias de aprendizaje en contextos informales. El caso de tres empresas de inserción", CIRIEC-España, Revista de Economía Pública, Social y Cooperativa, 94, 185-216, DOI: 10.7203/CIRIEC-E.94.12695.

CID, J. \& MARTÍ, J. (2012): "Turning points and returning points: Understanding the role of family ties in the process of desistance", European Journal of Criminology, 9 (6), 603-620. DOI: $10.1177 / 1477370812453102$.

COONEY, K. (2013): "Examining the labor market presence of US WISEs", Social Enterprise Journal, 9 (2), 147-163. DOI: 10.1108/SEJ-01-2013-0006.

COONEY, K. (2015): "Social Enterprise in the United States: WISEs and Other Worker-Focused Models", ICSEM Working Papers, 9. www.iap-socent.be/icsem-project.

DAVISTER, C., DEFOURNY, J. \& GREGOIRE, O. (2004): "Work Integration Social Enterprises in the European Union: An overview of existing models", Revue Internationale de l'Économie Sociale: Recma, 293, 24-50.

DOHERTY, B., HAUGH, H. \& LYON, F. (2014). "Social Enterprises as Hybrid Organizations: A Review and Research Agenda", International Journal of Management Reviews, 16 (4), 417-436. DOI: 10.1111/ijmr.12028.

ERAUT, M. (2009): Improving the Quality of Work Placements, AERA annual conference, http://learningtobeprofessional.pbworks.com/f/CHAPTER\%20D1.pdf.

ERAUT, M. \& HIRSH, W. (2007): The significance of workplace learning for indivi- duals, groups and organizations, SKOPE Monograph 6. Oxford: Oxford \& Cardiff Universities.www.skope.ox.ac.uk/ wp-content/uploads/2014/12/Monogrpah-09.pdf.

ERAUT, M., MAILLARDET, F.J., MILLER, C., STEADMAN, S., ALI, S., BLACKMAN, C. \& FURNER, J. (2004): Learning in the Professional Workplace: Relationships between Learning Factors and Contextual Factors, AERA Conference Paper, San Diego.

ERAUT, M., MAILLARDET, F.J., MILLER, C., STEADMAN, S., ALI, S., BLACKMAN, C., CABALLERO, C. (2005): What is Learned in the Workplace and How? Typologies and results from a cross-professional longitudinal study. Early Career Learning in the Professional Workplace. Paper presented at EARLI Conference, Nicosia.

EVANS, K., HODKINSON, P., RAINBIRD, H. \& UNWIN, L. (2006): Improving learning at work, London: Routledge.

FAEDEI (2018): Memoria Social Empresas de Inserción 2017, www.faedei.org

FAEDEI \& AERESS (2014): El acompañamiento en las empresas de inserción, www.faedei.org/images/docs/documento49.pdf 
FANTOVA, F. (2009): "Nota sobre acompañamiento social y autonomía personal", Políticas Sociales en Europa, 25, 167-175.

FERNÁNDEZ, A., GALARRETA, J. \& MARTíNEZ, N. (2007): Manual de Acompañamiento en las Empresas de Inserción: Proceso y herramientas, Bilbao: REAS.

GALLASTEGI, A. \& MARTínEZ, N. (2011): La Mejora de los Procesos de Acompañamiento en las Empresas de Inserción, Bilbao: Gizatea.

GLÉMAIN, P. (2018): "The Work Integration Social Enterprises as Learning Organizations: In the quest of a new local governance in order to build another model of local sustainable development", CIRIEC and BANCE, P. (dir.). Providing public goods and commons. Towards coproduction and new forms of governance for a revival of public action, 37-54. Liège: CIRIEC. DOI: 10.25518/ciriec. css1chap2.

GREGG, P. (2008): Realising Potential: A Vision for Personalised Conditionality and Support An independent report to the Department for Work and Pensions, www.dwp.gov.uk/welfare.

GUILLERA-MARCO, S. \& CHISVERT-TARAZONA, M.J. (Coord.) (2018): Acreditación de Competencias Profesionales. Guía para empresas de inserción, Madrid: Aeress y Faedei, https://box. freepresshost.com/public/054c49.

HILTROP, J.M. (1996): "Managing the changing psychological contract", Employee Relations. 18 (1), 36-48. www.angelfire.com/nb/ba1199/lesson028/pc1.pdf.

HIU-KWAN CHUI, C., Y SHUM, M.H. \& S LUM, T.Y. (2019): "Work integration social enterprises as vessels of empowerment? Perspectives from employees", Asia Pacific Journal Of Social Work and Development, 29 (2), 133-148. DOI: 10.1080/02185385.2018.1538899

KERLIN, J.A. (2013): "Defining Social Enterprise Across Different Contexts: A Conceptual Framework Based on Institutional Factors", Nonprofit and Voluntary Sector Quarterly, 42 (1), 84-108. DOI: 10.1177/0899764011433040.

LLINARES, L.I., ZACARÉS, J.J. \& CÓRDOBA, A.I. (2016): "Discussing employability: current perspectives and key elements from a bioecological model", Employee Relations, 38 (6), 961-974. DOI: 10.1108/ER-07-2015-0145.

MARHUENDA-FLUIXÁ, F. (2018): "Learning at work: Researching personal development and competence building in work integration companies", Educar, 54, 2, 491-509. DOI: 10.5565/rev/educar.801.

MARHUENDA-FLUIXÁ, F., BERNAD-GARCÍA, J.C. \& NAVAS-SAURIN, A. (2010): "Las prácticas en empresa como estrategia de enseñanza e inserción laboral: las empresas de inserción social", Revista de educación, 351, 139-161. 
MARSICK, V.J., \& WATKINS, K.E. (2001): "Informal and Incidental Learning", New Directions for Adult and Continuing Education, 89, 25-34.

MARTÍNEZ, I. \& MOLPECERES, M.A. (2010): "Lógicas de justificación en el sector asociativo", RETS 14, 17-40.

MARTíNEZ-RUEDA, N., GALARRETA, J. \& AROSTEGUI, I. (2018): "Factores organizacionales que inciden en la mejora de la empleabilidad de las Empresas de Inserción", CIRIEC-España, Revista de Economía Pública, Social y Cooperativa, 94, 123-154. DOI: 10.7203/CIRIEC-E.94.12697.

MARTíNEZ-RUEDA, N., GALARRETA, J. \& ARÓSTEGUI, I. (2018): "Empleabilidad y empresas de inserción: modelo de prestaciones y apoyos", Zerbitzuan, 67, 79-93. DOI: 10.5569/1134-7147.67.06.

MARTINEZ-RUEDA, N. \& GALARRETA, J. (2019): El acompañamiento en las Empresas de Inserción: Revisión y actualización del modelo de Gizatea, Bilbao: Gizatea

PÉREZ ERANSUS, B. (2004): "El acompañamiento social como herramienta de lucha contra la exclusión". Documentación Social, 135, 89-107.

PLANELLA, J. (2008): "Educación social, acompañamiento y vulnerabilidad : hacia una antropología de la convivencia", Revista Iberoamericana de Educación, 46 (5), 1-14.

QUINTÃO, C. (2007): "Empresas de inserción y empresas sociales en Europa", CIRIEC-España, Revista de Economía Pública, Social y Cooperativa, 59, 33-59.

SAN JOSÉ, P., ROCA, H. y CASTELLÓ, CH. (2019): "Acompañamiento en empresas de inserción", Noticias de la Economía Pública, Social y Cooperativa - Noticias del CIDEC, 60, 45-49.

SIIS (2011): Activación y derecho a la inclusión en el marco de las políticas de empleo y de garantía de ingresos en la CAPV, http://www.siis.net/documentos/ficha/197806.pdf. 
